\title{
Bending of electromagnetic wave in an ultra-strong magnetic field
}

\author{
Jin Young Kim \\ Department of Physics, Kunsan National University, Kunsan 573-701, Korea
}

\begin{abstract}
We consider the bending of light by nonlinear electrodynamics when the magnetic field $B$ exceeds the critical value $B_{\mathrm{c}}=m^{2} c^{2} / e \hbar=4.4 \times 10^{9} \mathrm{~T}$. Using the index of refraction derived from the analytic series representation in one-loop effective action of QED, we found the trajectory and the bending angle of light in geometric optics. The angle bent by ultra-strong magnetic field of magnetar was estimated and compared with the gravitational bending. The result may be useful in studying the lensing, birefringence, and other nonlinear quantum electrodynamic effects above $B_{\mathrm{c}}$.
\end{abstract}

PACS numbers: 12.20.Fv,41.20.Jb,95.30-k

*Electronic address: jykim@kunsan.ac.kr 
Electrodynamics is nonlinear not only in a continuous medium but also in a non-trivial vacuum by quantum correction. The vacuum polarization by virtual electron and positron in the presence of the non-trivial quantum electrodynamics (QED) vacuum causes the velocity shift and can be described by the index of refraction. For example, electromagnetic field can induce a material-like behavior by non-local features of the quantum vacuum structure. In the case of a weak field, the so-called Euler-Heisenberg effective Lagrangian [1-4] is a result of this quantum correction.

The fundamental scale for vacuum polarization is set by the critical field of QED, $B_{\mathrm{c}}=E_{\mathrm{c}} / c=m^{2} c^{2} / e \hbar=4.4 \times 10^{9} \mathrm{~T}$. Nonlinear properties by non-trivial QED vacua are being tested and confirmed with the advances in experimental devices, especially in high power laser technology [5-10]. However, as far as the phenomena related to the strong electromagnetic field are concerned, it is very difficult to test them in ground laboratories since the maximum available field is of the order $B \sim 10^{2} \mathrm{~T}$. One can think of the astrophysical objects as sources of the strong electromagnetic field. A significant growth in this field of research has been done due to the observation of strong magnetic field in neutron star [11 24].

The light bending by a massive object is a result of general relativity and is a useful tool in astrophysics. The light bending can also happen when the light ray passes electrically or magnetically charged objects by the nonlinear interaction of QED. In geometric optics, light path can be bent by a continually varying index of refraction. If there is a gradient in the index of refraction by non-trivial QED vacuum, one can calculate the bending angle in analogy with the geometric optics. For example, Denisov et al. [13] calculated the bending angle using the eikonal equation when a ray is passing through the equator of the magnetic dipole. In the previous work of the author [23, the trajectory of light and bending angle in a Coulombic field were computed in geometric optics based on the Euler-Heisenberg effective action. Also the bending angle of light by the electric field of a charged black hole was estimated and a general formula of the bending angle valid for any orientation of the magnetic dipole was derived [24].

In astrophysics there are objects with very strong electric or magnetic field. As far as the magnetic field is concerned, there are neutron stars with the surface magnetic field above the QED critical field, known as magnetars. Since the Euler-Heisenberg effective action is represented as an asymptotic series, its application is confined to the weak field limit. So 
the result based on the Euler-Heisenberg Lagrangian is not valid in this ultra-strong field limit. The knowledge on the effective action for strong field limit is crucial to study the nonlinear electromagnetic properties above the critical field $B_{\mathrm{c}}$. In this paper, we will study the bending of light in the ultra-strong magnetic field of magnetars.

To study the light bending above the critical field, we need the complete expression of the action. Recently, analytic series representation for the one-loop effective action of QED has been studied from Schwinger's integral form [25 30]. When the electric or magnetic field is above the QED critical limit, light propagating in such ultra-strong electromagnetic fields no longer satisfied the light cone condition, i.e., $v \neq c$. We use the explicit expression for the average velocity of light in ultra-strong electric and magnetic fields derived by Cho et al. [29]

$$
\begin{aligned}
& \bar{v}_{\mathrm{m}}^{2} \simeq \frac{1-\frac{e^{2}}{12 \pi^{2}}\left(\ln \frac{e B}{m^{2}}+c_{1}+1\right)}{1-\frac{e^{2}}{12 \pi^{2}}\left(\ln \frac{e B}{m^{2}}+c_{2}\right)+\frac{e^{3} B}{24 \pi^{2} m^{2}}} \\
& \bar{v}_{\mathrm{e}}^{2} \simeq \frac{1-\frac{e^{2}}{12 \pi^{2}}\left(\ln \frac{e E}{m^{2}}+c_{2}\right)}{1-\frac{e^{2}}{12 \pi^{2}}\left(\ln \frac{e E}{m^{2}}+c_{1}+1\right)}
\end{aligned}
$$

where

$$
\begin{aligned}
& c_{1}=-\gamma-\ln \pi+\frac{6}{\pi^{2}} \zeta^{\prime}(2)=-2.29191 \ldots, \\
& c_{2}=\ln 2 \pi+c_{1}+\frac{3}{2}-\gamma=-1.82070 \ldots
\end{aligned}
$$

The corresponding index of refraction is given by $n=1 / v$. Up to the leading order in one-loop, one can approximate the index of refraction $n$ by

$$
\begin{aligned}
& \bar{n}_{\mathrm{m}} \simeq \sqrt{1+\frac{e^{2}}{12 \pi^{2}}\left(c_{1}-c_{2}+1\right)+\frac{e^{3} B}{24 \pi^{2} m^{2}}}, \\
& \bar{n}_{\mathrm{e}} \simeq \sqrt{1-\frac{e^{2}}{12 \pi^{2}}\left(c_{1}-c_{2}+1\right)} .
\end{aligned}
$$

Now we consider the bending of light when it passes near the astronomical object with strong electric or magnetic field above the critical value. As a source of ultra-strong electric field one can think of a charged black hole theoretically. As a source of ultra-strong magnetic field one can consider the magnetar whose magnetic field strength on the surface is estimated up the order of $10^{11} \mathrm{~T}$. The physical limit to the magnetic field of neutron star is of the order $10^{12}-10^{14} \mathrm{~T}[31,32]$. Beyond this limit, the fluid inside the star would mix and the magnetic field would dissipate. No objects in the universe can maintain fields stronger than this limit. 


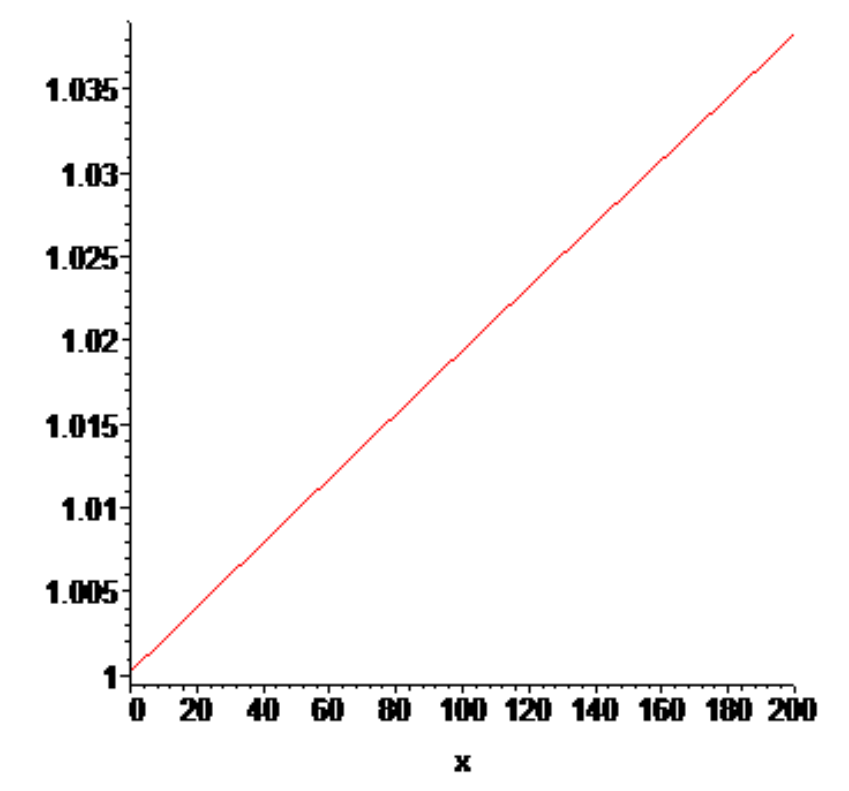

FIG. 1: Index of refraction $\bar{n}_{\mathrm{m}}$ plotted as a function of $x=B / B_{\mathrm{c}}$ up to $x=200\left(\sim 10^{12} / 4.4 \times 10^{9}\right)$.

Thus we consider the maximal field strength up to $10^{12} \mathrm{~T}$. Note that the index of refraction is close to one for the range of our consideration (see figure 1).

Eq. (4) tells us that there is no significant change of refraction index by ultra-strong electric field. So it is expected that the bending of light is negligible when photons pass the ultra-strong electric field of a charged black hole. To calculate the bending angle by the ultra-strong magnetic field of magnetar, we consider the trajectory of a light ray when it passes the magnetic field of a magnetic dipole. In general the bending angle will depend on both the orientation of the dipole relative to the direction of the incoming ray and the polarization of the light. To simplify the calculation, we consider the case when the photon path is perpendicular to the dipole moment and traveling on the equator of the dipole. The generalization for arbitrary orientation of the dipole axis can be straightforward as in [24]. We consider the magnetic dipole located at the origin directing along the $z$ axis and the incident photon is coming from $x=-\infty$ with an impact parameter $b$ (see figure 2).

Because the quantum correction in the presence of ultra-strong magnetic field is represented in terms of index of refraction, we can consider the analogous light propagation in a classical medium within geometric optics formalism. From the Snell's law, the trajectory equation can be written as [23, 24]

$$
\frac{d \mathbf{u}}{d s}=\frac{1}{n}(\mathbf{u} \times \nabla n) \times \mathbf{u},
$$




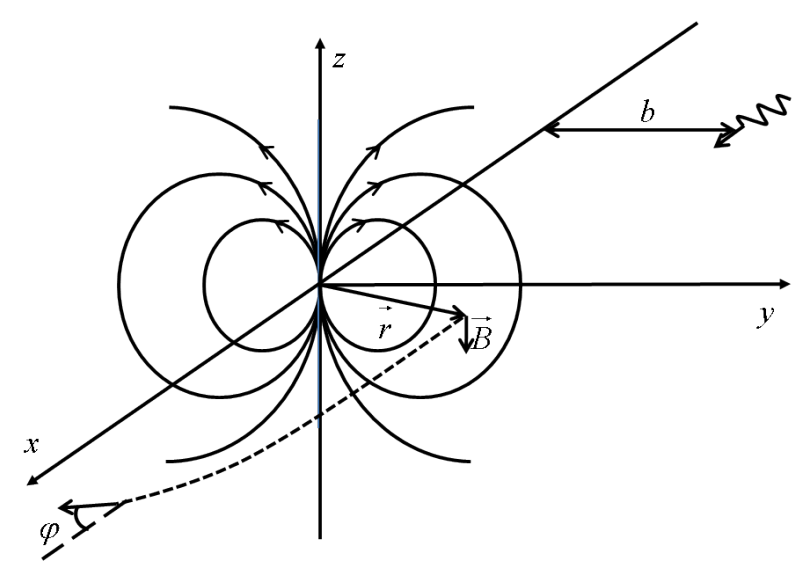

FIG. 2: Schematic of light bending by magnetic dipole when the photon path is on the equator of the dipole.

where $\mathbf{u}$ denotes the unit vector in the direction of propagation, $s$ denotes the distance parameter of the light trajectory with $d s=|d \overrightarrow{\mathbf{r}}|$, and

$$
\mathbf{u}=\frac{d \overrightarrow{\mathbf{r}}}{d s}
$$

When the correction to the index of refraction is small, the trajectory equation can be approximated to the leading order as

$$
\frac{d \mathbf{u}}{d s}=\left(\mathbf{u}_{0} \times \nabla n\right) \times \mathbf{u}_{0}
$$

where $\mathbf{u}_{0}$ denotes the initial direction of the incoming photon. For a photon coming in from $x=-\infty$ and moves to $+x$ direction,

$$
\mathbf{u}_{0}=(1,0,0)
$$

and defining $\nabla n \equiv\left(\eta_{1}, \eta_{2}, \eta_{3}\right)$ the trajectory equation becomes

$$
\frac{d^{2} x}{d s^{2}}=0, \quad \frac{d^{2} y}{d s^{2}}=\eta_{2}, \quad \frac{d^{2} z}{d s^{2}}=\eta_{3}
$$

The first equation shows that $d s=d x$ at the leading order and the trajectory equations for $y(x)$ and $z(x)$ are given by

$$
\frac{d^{2} y}{d x^{2}}=\eta_{2}, \quad \frac{d^{2} z}{d x^{2}}=\eta_{3},
$$

which will be used in the following analysis. Note that, on the equator of the dipole, there is no gradient in $z$ direction by symmetry, $\eta_{3}=0$. Thus there is no bending in $z$ direction and the bending will occur toward the center of the dipole. 
The magnetic field at a distance $r$ on the equator of the magnetic dipole is given by

$$
\mathrm{B}=B_{0} \frac{r_{0}^{3}}{r^{3}}(-\hat{z}),
$$

where $B_{0}$ is the magnetic field on the surface of the neutron star. Restoring the physical constants, the index of refraction can be written as

$$
\bar{n}_{\mathrm{m}} \simeq \sqrt{1+\frac{\alpha}{3 \pi}\left(c_{1}-c_{2}+1\right)+\frac{\alpha}{6 \pi} \frac{B_{0}}{B_{\mathrm{c}}}\left(\frac{r_{0}}{r}\right)^{3}},
$$

where $\alpha=1 / 137$ is the fine structure constant. As shown in figure 1 , the index of refraction does not deviate much from one up to $B_{0}=10^{12} \mathrm{~T}$. Thus, we approximate the index of refraction as

$$
\bar{n}_{\mathrm{m}} \simeq 1+\frac{\alpha}{6 \pi}\left(c_{1}-c_{2}+1\right)+\frac{\alpha}{12 \pi} \frac{B_{0}}{B_{\mathrm{c}}}\left(\frac{r_{0}}{r}\right)^{3},
$$

and the trajectory equation can be written explicitly as

$$
\frac{d^{2} y}{d x^{2}}=\eta_{2}=-\frac{\alpha}{4 \pi} \frac{B_{0} r_{0}^{3}}{B_{\mathrm{c}}} \frac{y}{r^{5}} .
$$

For the incoming photon with the impact parameter $b$, the initial condition reads

$$
y(-\infty)=b, \quad y^{\prime}(-\infty)=0 .
$$

Integrating Eq. (14) by putting $y=b$ for the leading order solution, we obtain

$$
\begin{aligned}
y^{\prime}(x) & =-\frac{\alpha}{12 \pi} \frac{B_{0}}{B_{\mathrm{c}}} \frac{r_{0}^{3}}{b^{3}}\left[\frac{x}{\sqrt{b^{2}+x^{2}}}\left(\frac{b^{2}}{b^{2}+x^{2}}+2\right)+2\right], \\
y(x) & =b\left[1-\frac{\alpha}{12 \pi} \frac{B_{0}}{B_{\mathrm{c}}} \frac{r_{0}^{3}}{b^{3}}\left(2 \frac{\sqrt{b^{2}+x^{2}}+x}{b}-\frac{b}{\sqrt{b^{2}+x^{2}}}\right)\right] .
\end{aligned}
$$

The total bending angle $\varphi_{\mathrm{m}}$ can be obtained from $y^{\prime}(\infty)$,

$$
\left|y^{\prime}(\infty)\right|=\tan \varphi_{\mathrm{m}} \simeq \varphi_{\mathrm{m}},
$$

and is given by

$$
\varphi_{\mathrm{m}}=\frac{\alpha}{3 \pi} \frac{B_{0}}{B_{\mathrm{c}}} \frac{r_{0}^{3}}{b^{3}} .
$$

Compared with the bending angle obtained from the asymptotic form of Euler-Heisenberg effective action valid in the weak field limit [13, 24],

$$
\varphi_{\mathrm{mEH}} \propto \frac{B_{0}^{2}}{b^{6}},
$$


the power dependence $\varphi_{\mathrm{m}} \propto B_{0} / b^{3}$ implies that the magnetic bending may be important at short distance. Let us estimate the bending angle for an ultra-strongly magnetized neutron star and compare it with the gravitational bending. According to the general relativity, the gravitational bending angle is given by

$$
\varphi_{\mathrm{g}}=\frac{4 G \mathcal{M}}{b c^{2}} .
$$

Consider a neutron star with mass $\mathcal{M}=\mathcal{M}_{\text {sun }}=2 \times 10^{30} \mathrm{~kg}$ and radius $r_{0}=10 \mathrm{~km}$. Parameterizing the impact parameter in units of the radius $b=\zeta r_{0}$ with $\zeta>1$, the bending by a ultra-strong magnetic field can be expredded as

$$
\varphi_{\mathrm{m}}=\frac{\alpha}{3 \pi} \frac{B_{0}}{B_{c}} \frac{1}{\zeta^{3}} .
$$

The maximal gravitational bending for $\zeta=1\left(b=r_{0}\right)$ is $\varphi_{\mathrm{g}}=0.59 \mathrm{rad}$. For a magnetar with the field strength on the surface $B_{0}=10^{11} \mathrm{~T}$, the maximal value of the magnetic bending angle is estimated as $\varphi_{\mathrm{m}}=1.8 \times 10^{-2} \mathrm{rad}$. This value is one order of magnitude smaller than the gravitational bending. For $B_{0}=10^{12} \mathrm{~T}$, the magnetic bending can be comparable to the gravitational bending.

So far we have considered the light bending by average value of the index of refraction. In the presence of strong magnetic field, QED renders the vacuum birefringent; the light velocities for perpendicular and parallel modes are different. The asymptotic formula for the light velocities for the ultra-strong field limit are given by [29]

$$
\begin{aligned}
& v_{\perp}^{2} \simeq \frac{1-\frac{e^{2}}{1 \pi^{2}}\left(\ln \frac{e B}{m^{2}}+c_{1}+\frac{3}{2}\right)}{1-\frac{e^{2}}{12^{2}}\left(\ln \frac{e B}{m^{2}}+c_{1}+\frac{1}{2}\right)}, \\
& v_{\|}^{2} \simeq \frac{1-\frac{e^{2}}{12 \pi^{2}}\left(\ln \frac{e B}{m^{2}}+c_{1}+\frac{1}{2}\right)}{1-\frac{e^{2}}{12 \pi^{2}}\left(\ln \frac{2 e B}{\pi m^{2}}+1-\gamma\right)+\frac{e^{3} B}{12 \pi^{2} m^{2}}} .
\end{aligned}
$$

The corresponding leading order terms of the indices of refraction are

$$
\begin{aligned}
& n_{\perp} \simeq 1+\frac{e^{2}}{24 \pi^{2}}, \\
& n_{\|} \simeq 1+\frac{e^{2}}{24 \pi^{2}}\left(c_{1}-\frac{1}{2}+\ln \frac{\pi}{2}+\gamma\right)+\frac{e^{3} B}{24 \pi^{2} m^{2}} .
\end{aligned}
$$

Compared with Eq. (3), the bending angle for the parallel mode will be twice the value given by Eq. 19 while there is no bending for the perpendicular mode. This birefringence effect can be one way of testing the validity of our calculation if the allowed precision in measuring the photon polarization is enough. 
Note that, from Eqs. (19) and (21), the relative bending $\varphi_{\mathrm{m}} / \varphi_{\mathrm{g}} \propto 1 / b^{2}$. For example, at impact parameter $b=10 r_{0}$, the magnetic bending $\varphi_{\mathrm{m}}=1.8 \times 10^{-5} \mathrm{rad}$ is three orders of magnitude smaller then the gravitational bending $\varphi_{\mathrm{g}}=5.9 \times 10^{-2} \mathrm{rad}$. Even if the magnetic bending angle may be small compared with the gravitational bending, one can check the result in this work by measuring the total bending angles for different values of the impact parameter. Since the power dependence of two bending angles on the impact parameter is different, $\varphi_{\mathrm{g}} \propto 1 / b, \varphi_{\mathrm{m}} \propto 1 / b^{3}$, one may test the power dependence by fitting the data from different impact parameters to $\varphi_{\text {tot }}=\varphi_{\mathrm{g}}+\varphi_{\mathrm{m}}$.

The constraint on strong B-field consistent with the one-loop approximation is $B / B_{\mathrm{c}}<$ $\pi / \alpha \simeq 430$. The maximum field strength that we considered in this work, $B \sim 10^{12} \mathrm{~T}$ ( $\left.x=B / B_{\mathrm{c}} \simeq 200\right)$, is within this range. So our result may be useful in studying the lensing, birefringence, and other nonlinear quantum electrodynamic effects by ultra-strong magnetic field of magnetars.

\section{Acknowledgments}

We would like to thank M. I. Park for discussion and help. This research was supported by Basic Science Research Program through the National Research Foundation of Korea (NRF) funded by the Ministry of Education, Science and Technology (12A12840581).

[1] W. Heisenberg and H. Euler, Z. Phys. 98, 714 (1936).

[2] J. S. Schwinger, Phys. Rev. 82, 664 (1951).

[3] Z. Bialynicka-Birula and I. Bialynicka-Birula, Phys. Rev. D 2, 2341 (1970).

[4] S. L. Adler, Annals Phys. 67, 599 (1971).

[5] S. K. Lamoreaux, Phys. Rev. Lett. 78, 5 (1997).

[6] D. L. Burke, et al., Phys. Rev. Lett. 79, 1626 (1997).

[7] A. Di Piazza, K. Z. Hatsagortsyan, and C. H. Keitel, Phys. Rev. Lett. 97, 083603 (2006).

[8] B. King, A. Di Piazza, and C. H. Keitel, Nature Photon. 4, 92 (2010).

[9] W. J. Kim, A. O. Sushkov, D. A. R. Dalvit, and S. K. Larmoreaux, Phys. Rev. Lett. 103, 060401 (2009). 
[10] A. O. Sushkov, W. J. Kim, D. A. R. Dalvit, and S. K. Larmoreaux, Nature Phys. 7, 230 (2011).

[11] V. A. De Lorenci, N. Figueredo, H. H. Fliche, and M. Novello, Astron. Astrophys. 369, 690 (2001).

[12] N. J. Shaviv, J. S. Heyl, and Y. Lithwick, Mon. Not. R. Astron. Soc. 306, 333 (1999).

[13] V. I. Denisov, I. P. Denisova, and S. I. Svertilov, Dokl. Akad. Nauk. Ser. Fiz. 380, 435 (2001).

[14] V. I. Denisov, I. P. Denisova, and S. I. Svertilov, Dokl. Phys. 46, 705 (2001).

[15] J. S. Heyl and N. J. Shaviv, Phys. Rev. D 66, 023002 (2002).

[16] V. I. Denisov and S. I. Svertilov, Astron. Astrophys. 399, L39 (2003).

[17] J. S. Heyl, N. J. Shaviv, and D. Lloyd, Mon. Not. R. Astron. Soc. 342, 134 (2003).

[18] V. I. Denisov, I. P. Denisova, and S. I. Svertilov, Theor. Math. Phys. 140, 1001 (2004).

[19] V. I. Denisov and S. I. Svertilov, Phys. Rev. D71, 063002 (2005).

[20] A. Dupays, C. Robilliard, C. Rizzo, and G. F. Bignami, Phys. Rev. Lett. 94, 161101 (2005).

[21] P. A. Vshivtseva, V. I. Denisov, and I. V. Krivchenkov, Theor. Math. Phys. 150, 73 (2007).

[22] D. Mazur and J. S. Heyl, Mon. Not. R. Astron. Soc. 412, 1381 (2011).

[23] J. Y. Kim and T. Lee, Mod. Phys. Lett. A 26, 1481 (2011).

[24] J. Y. Kim and T. Lee, JCAP 1111, 017 (2011).

[25] J. S. Heyl and L. Hernquist, Phys. Rev. D 55, 2449 (1997).

[26] J. S. Heyl and L. Hernquist, J. Phys. A 30, 6485 (1997).

[27] W. Dittrich and H. Gies, Phys. Rev. D 58, 025004 (1998).

[28] Y. M. Cho and D. G. Pak, Phys. Rev. Lett. 86, 1947 (2001).

[29] Y. M. Cho, D. G. Pak, and M. L. Walker, Phys. Rev. D 73, 065014 (2006).

[30] S.-W. Hu and B.-B. Liu, J. Phys. A: Math. Theor. 40, 13859 (2007).

[31] W. T. Zaumen, Astrophys. J. 210, 776 (1976).

[32] I. Lerche and D. M. Shramm, Astrophys. J. 216, 881 (1977). 